\title{
CISTOADENOMA MUCINOSO HEPÁTICO
}

\author{
HEPATIC MUCINOUS CYSTADENOMA \\ Héctor U. Juárez-Granda1,c, Consuelo E. Cornejo-Carrasco 1,2,a, , Wilder E. Ramos-Castillo ${ }^{1,6}$
}

\begin{abstract}
RESUMEN
El cistoadenoma hepático es una neoplasia muy rara, con menos de 250 casos reportados en la literatura mundial, que se da con mayor frecuencia en mujeres. Por la dificultad de diferenciar el quiste simple y el quiste hidatídico con el cistoadenoma hepático; así como su recidiva y posibilidad de displasia o carcinoma, si no es tratado mediante resección completa, es que presentamos el caso de una mujer de 56 años con historia de quiste hepático simple tratado en 2 oportunidades con destechamiento simple y que recidivaron, ya que se trataba de un cistoadenoma hepático.
\end{abstract}

Palabras clave: Cistoadenoma hepático; Cistoadenocarcinoma hepático; Estroma ovárico; Tomografía (TC); Resonancia magnética nuclear (RMN). (fuente: DeCS BIREME)

\begin{abstract}
Hepatic cystadenoma is a very rare neoplasm, with less than 250 cases reported in the world literature, which occurs more frequently in women. Due to the difficulty of differentiating the simple cyst and the hydatid cyst with the hepatic cystadenoma; as well as its recurrence and possibility of dysplasia or carcinoma, if it is not treated by complete resection, we present the case of a woman of 56 years old with history of simple hepatic cyst treated on 2 occasions with simple hepatic cyst unroofing and that they relapsed, so it was a hepatic cystadenoma.
\end{abstract}

Key words: Hepatic cystadenoma; Hepatic cystadenocarcinoma; Ovarian stroma; Tomography (CT); Magnetic resonance imaging (MRI). (source: MeSH NLM)

\section{INTRODUCCIÓN}

La enfermedad quística del hígado ocurre entre el 3 al $20 \%$ de la población, cuya incidencia aumenta con la edad; siendo el quiste simple la patología más común ${ }^{1,2}$. En 1887 Heuter describió el primer reporte de cistoadenoma hepático, que ahora representan menos del $5 \%$ de todas las lesiones quísticas del hígado $^{2,3}$ y están comprendidas dentro de las neoplasias quísticas del hígado. Es más frecuente en mujeres, y en más del $85 \%$ se diagnostica entre la cuarta y sexta década de la vida4.

Estas lesiones son derivadas del epitelio biliar que histológicamente están compuestas por tres capas: Una capa externa de colágeno que contiene vasos sanguíneos y nervios; una capa estromal "tipo ovárico", denso que contiene células de músculo además de tejido adiposo y una capa epitelial columnar (a veces cúbica secretora de mucina) (5, $^{5}$. La característica más llamativa de este tumor quístico es la presencia de estroma de tipo ovárico debajo del epitelio del revestimiento quístico ${ }^{5}$.

Es importante destacar que el epitelio puede mostrar displasia de alto grado y progresar a carcinoma desde 20 a $30 \%$ de los casos $^{2-6}$. Sin embargo, en la mayoría de las series, los cistoadenomas hepáticos conllevan un riesgo extremadamente bajo de morbilidad y mortalidad después de una resección quirúrgica adecuada7.

Se describen patológicamente dos tipos de cistoadenomas hepáticos: Mucinosos y serosos.

\footnotetext{
${ }^{1}$ Hospital Nacional Edgardo Rebagliati Martins, Lima-Perú.

${ }^{2}$ Facultad de Medicina Humana,Universidad Ricardo Palma, Lima-Perú.

${ }^{a}$ Médico Asistente de Cirugía General y Digestiva.

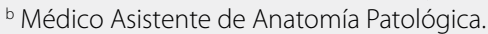

'Médico Residente de Cirugía General y Digestiva.

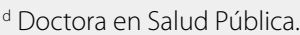

Citar como: Héctor U. Juárez-Granda, Consuelo E. Cornejo-Carrasco, Wilder E. Ramos-Castillo. Cistoadenoma mucinoso hepático. [Caso Clínico].2019;19(2):00-00. (Abril 2019). DOl 10.25176/RFMH.v19.n2.2075 
El cistoadenoma mucinoso es el tipo predominante (95\% de los casos) que se presenta en las mujeres ${ }^{1-7}$. Estas neoplasias se encuentran con mayor frecuencia dentro del lóbulo derecho del hígado (55\%), pero también pueden afectar el lóbulo izquierdo (29\%) o ambos lóbulos (16\%) y varía de 1.5 a $35 \mathrm{~cm}$ de diámetro y puede pesar hasta 6000 gramos $^{6-8,10}$.

Tradicionalmente, se cree que los cistoadenomas se originan del epitelio biliar, posiblemente en un conducto biliar congénitamente aberrante. Otras posibilidades son que los tumores surgen directamente de células embrionarias o células endocrinas peribiliares ${ }^{8}$.

Los principales diagnósticos diferenciales incluyen: quiste hepático simple, cistoadenocarcinoma hepático, enfermedad de Caroli, neoplasia mucinosa papilar de ducto biliar, quiste hidatídico, quiste hepático post traumático, enfermedad poliquística hepática, absceso hepático ${ }^{5,8,10,11}$. Al poder confundirlos con quistes simples o quistes hidatídicos por su mayor prevalencia, podríamos dar un tratamiento inadecuado a esta patología, ya que en ellas sería suficiente con un destechamiento del quiste, pero en un cistoadenoma hepático se debe realizar la resección quirúrgica completa del mismo, ya que recidivan si se deja tejido residual, e incluso solo con el estudio de anatomía patológica de toda la tumoración quística se puede descartar un cistoadenocarcinoma. Ante la importancia de diferenciar esta neoplasia quística hepática de las otras enfermedades quísticas del hígado, es que presentamos el presente caso clínico.

\section{REPORTE DE CASO}

Mujer de 52 años de edad, natural y procedente de Lima, sin antecedentes médicos de importancia, con antecedentes quirúrgicos de destechamiento de quiste simple laparoscópico en el 2002, cuyo informe de anatomía patológica fue quiste simple seroso y en el 2007 una laparotomía exploratoria + destechamiento de quiste simple hepático recidivado + colecistectomía con resultado de anatomía patológica como quiste simple hepático con fibrosis de pared. En enero del 2018 acude a consultorio externo por presentar dolor abdominal tipo cólico en hipocondrio derecho de baja intensidad en los 4 últimos meses, por lo que se realiza una TC sin contraste (la paciente era alérgica al yodo), evidenciándose una lesión hipodensa multiloculada en segmento IV A, IV B y V de $130 \times 80 \times 70 \mathrm{~mm}$. No calcificaciones significativas, sin grandes vesículas en su interior, ni dilatación de las vías biliares.

Es referida a nuestro hospital y se le hospitaliza en abril del 2018, donde se realiza una nueva TC abdominal en donde se observa una extensa formación quística loculada que ocupa los segmentos hepáticos IVA y IVB de $119 \times 75$ mm con finos tabiques y fina calcificación en la pared. Adicionalmente otra formación quística en el segmento $V$ de $30 \times 20$ mm, además de una formación quística en su interior a manera de "vesícula". Además de otras lesiones quísticas en su aspecto cefálico y lateral izquierdo. (Figura 1. A, B, C, D).

La resonancia magnética abdominal mostró una gran imagen quística multiloculada ubicada en casi todo el lóbulo izquierdo de 126 × 80 mm, esta lesión desplaza los trayectos vasculares sin infiltrarlos. (Figura 1. E, F).

Sus exámenes de laboratorio como hemograma, perfil hepático, bioquímica y marcadores tumorales (CEA y Ca 19.9) fueron normales.

La paciente es operada con el diagnostico preoperatorio de tumoración quística hepática recidivada: Cistoadenoma versus cistoadenocarcinoma. Se le realiza una periquistectomía + hepatectomía izquierda + resección parcial del segmento $\mathrm{V}$ + liberación de adherencias; encontrándose múltiples adherencias firmes de epiplón, duodeno y asa delgada a pared de quiste, el cual involucra los segmentos II, III, IVA, IVB y parcialmente el $V$ de aproximadamente $130 \mathrm{x}$ $80 \mathrm{~mm}$, con pared blanquecina de $3 \mathrm{~mm}$ de espesor conteniendo líquido levemente amarillento y algo viscoso en su interior y contactando íntimamente con la vía biliar principal y rama hepática izquierda. (Figura 2. $G, H)$.

El informe de anatomía patológica refiere que recibe un producto de hepatectomia + periquistectomía: Hígado con quiste hepático de $120 \times 90 \times 25 \mathrm{~mm}$ adyacente a segmento III, con contenido mucinoso en moderada cantidad, pared de $3 \mathrm{~mm}$ con múltiples estructuras quísticas de $7 \mathrm{~mm}$. Presenta epitelio mucinoso y estroma ovárico. Diagnóstico: Cistoadenoma mucinoso sin displasia ni componente invasivo, bordes quirúrgicos libres de lesión. (Figura 2. I, J).

La paciente tiene buena evolución y salió de alta a los 7 días del postoperatorio, En el seguimiento la paciente está asintomática y no hay recidiva de la enfermedad a los 9 meses de la cirugía. 

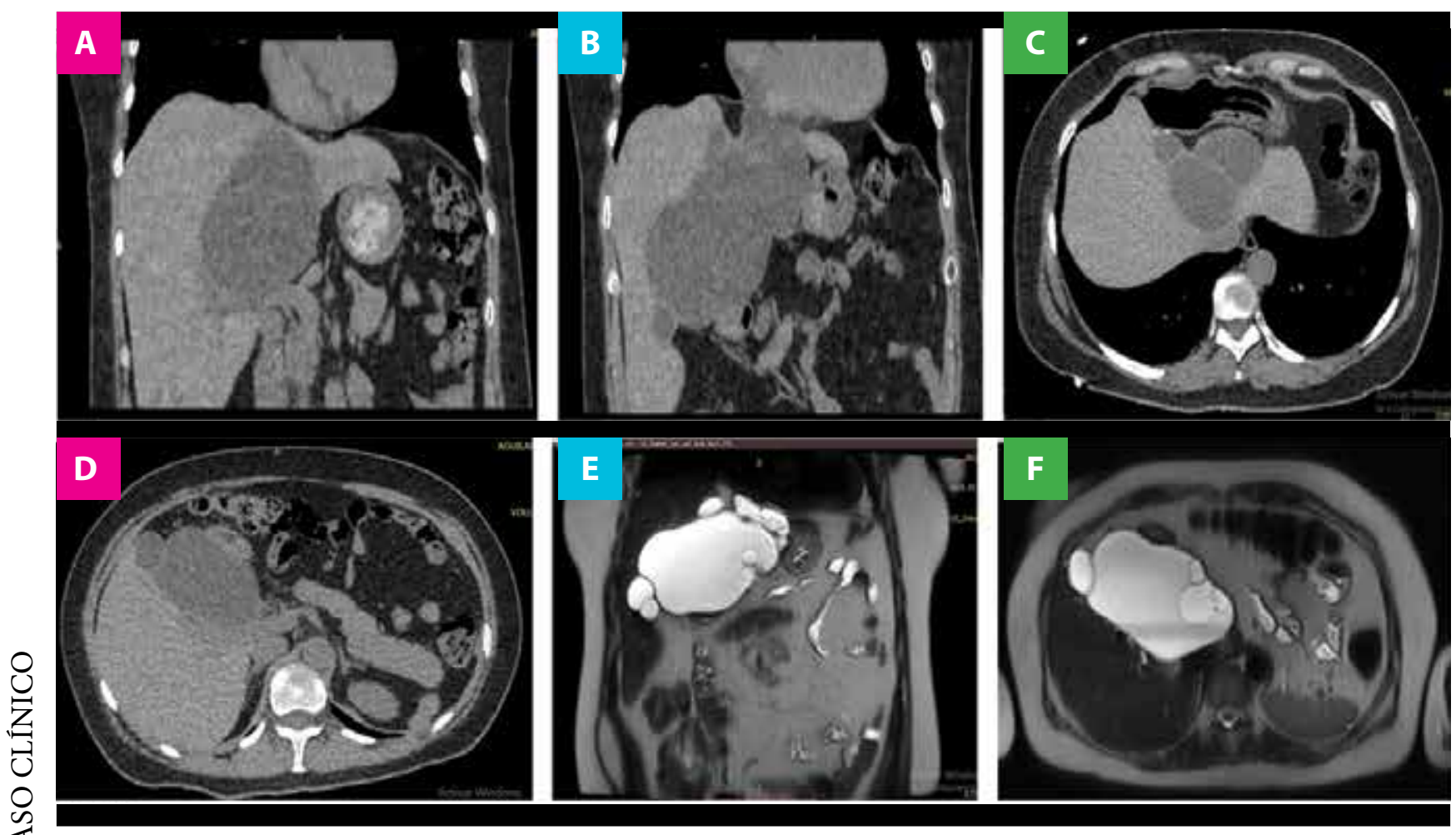

Figura 1. Tomografía $(A, B, C, D)$. Resonancia Magnética Nuclear $(E, F)$

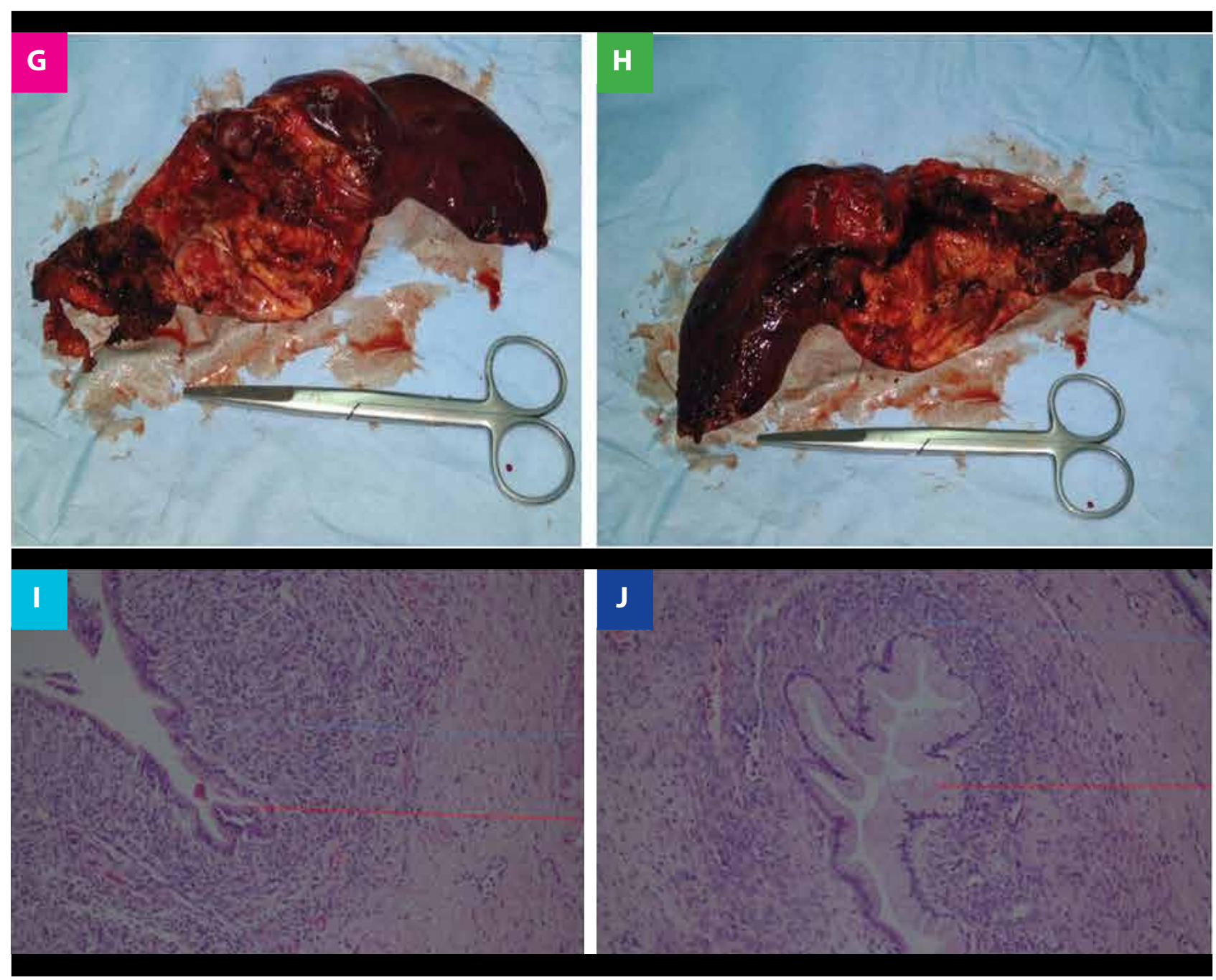

Figura 2. Pieza operatoria del hígado izquierdo y parcial segmento 5 con cistoadenoma (G, H). Microscopia: Epitelio mucinoso señalado con flecha roja y el estroma ovárico señalado con flecha azul $(\mathrm{I}, \mathrm{J})$. 


\section{DISCUSIÓN}

El cistoadenoma hepático es una enfermedad quística benigna poco frecuente, con menos de 250 casos reportados en la literatura mundial ${ }^{2}$.

Aparecen como lesiones quísticas multiloculares hasta en $84 \%$ de los casos, (rara vez uniloculares) y están rodeadas por una cápsula fibrosa lisa y gruesa. Éstos tumores contienen numerosos tabiques internos y proyecciones papilares intraluminales, que están revestidos por un epitelio biliar cuboidal o cilíndrico secretor de moco con un estroma ovárico subepitelial, descrito como patognomónico por Edmondson en $1958^{2,5,12-15}$.

Las conexiones verdaderas con el árbol biliar son raras. Sin embargo, el $50 \%$ de los de los quistes mucinosos contienen células endocrinas, lo que sugiere que pueden originarse en las glándulas peribiliares intrahepáticas ${ }^{6,8}$.

La presentación clínica puede variar considerablemente, muchos pacientes son asintomáticos hasta un $14 \%$; otros presentan síntomas inespecíficos, más comúnmente dolor abdominal, distensión y masa palpable $(55 \% \text { a } 90 \%)^{2,8,10,12}$.

Aunque los valores de laboratorio son normales en la mayoría de los pacientes, aproximadamente el 20\% presentan pruebas de función hepática alterada, como un nivel anormal de bilirrubina y GGT por compresión de la vía biliar, originando ictericia obstructiva y colangitis, lo que raramente se presenta ${ }^{8}$.

Los estudios inmunohistoquímicos que incluyen el receptor de estrógeno (positivos hasta en un 60-100\%), el receptor de progesterona y la inhibina son útiles para resaltar el estroma de tipo ovárico y establecer el diagnóstico, especialmente en los casos con estroma hialinizado. Los estudios inmunohistoquímicos para citoqueratina 7 (CK7), CK8, CK18, CK19, CA-19-9 y AE1 / AE3 resaltan el epitelio $0^{5,8,14}$.

En cuanto a marcadores tumorales se ha visto que el CA19.9 y CEA séricos aumentan en algunos tipos de tumores quísticos mucinosos, más no es un hallazgo universal y por lo tanto no debe ser usado como parámetro diagnóstico ${ }^{16}$, estos mismos marcadores tumorales obtenidos a través de la punción del quiste dan una sensibilidad y especificidad de $100 \%$ y $94 \%$ respectivamente sin embargo hay riesgo de diseminación peritoneal y pleural8, por lo que no se debe realizar.

Dentro de los exámenes de imágenes, el aspecto característico de la TC es una masa quística compleja solitaria con una cápsula fibrosa gruesa bien definida, con algunas calcificaciones en sus paredes, tabiques internos y nodularidad mural, llegando a tener una sensibilidad de $87 \%$ y una especificidad del $100 \%{ }^{6,15}$. La diferencia clave entre cistoadenoma o cistoadenocarcinoma hepático y un quiste hepático hemorrágico o infectado es que la cápsula, los tabiques internos y los nódulos murales muestran realce de contraste en el primero y no en el segundo. En general los nódulos o pólipos murales y las excrecencias pedunculadas son más frecuentes en el cistoadenocarcinoma biliar que en el cistoadenoma biliar, sin embargo, se han observado nódulos murales en los cistoadenomas biliares sin evidencia de neoplasia maligna en patología 25,10 .

En la RM, un cistoadenoma biliar no complicado aparece como una masa quística multilocular, con una intensidad de señal baja homogénea en imágenes ponderadas en $\mathrm{T} 1$ y alta intensidad de señal homogénea en imágenes ponderadas en $\mathrm{T} 2^{9,10}$.

En relación al tratamiento: La aspiración, esclerosis, drenaje interno, marsupialización o resección parcial con o sin ablación de la cavidad pueden dar lugar a una tasa de recurrencia de hasta 90 a 100\% ${ }^{2,13}$. Debido a la alta tasa de recidiva con resecciones parciales y ante la dificultad de diferenciar el cistoadenoma del cistoadenocarcinoma hepático en el pre-operatorio, ya que el estudio patológico de todo el quiste es el único que puede descartar displasia y carcinoma es que se recomienda la resección quirúrgica radical con márgenes negativos; como se hizo en el caso presentado.

Es importante tener en consideración esta patología, para no confundirlo con otras enfermedades quísticas del hígado, así como se dio en este caso que fue tratado 2 veces como quiste simple y recidivó, ya que se trataba de un cistoadenoma; por lo tanto, hay que realizar un buen estudio clínico, de imágenes y de anatomía patológica.

Contribuciones de autoría: Los autores participaron en la generación, redacción y aprobación final del caso clínico.

Financiamiento: Autofinanciado.

Conflicto de interés: Los autores declaran no tener conflicto de interés en la publicación de este casi clínico.

Recibido: 26 de octubre de 2018

Aprobado: 27 de enero de 2019

Correspondencia: Consuelo Elsa Cornejo Carrasco

Dirección: El cortijo 473 casa Nº 8 Urb. Monterrico Chico. Santiago de Surco, Lima - Perú.

Celular: +51998451040

Correo:docconsuelocornejo@gmail.com 


\section{REFERENCIAS BIBLIOGRÁFICAS}

1.- Marrero J, Ahn J , Rajender R. ACG Clinical Guideline: The Diagnosis and Management of Focal Liver Lesions. Am J Gastroenterol. 2014; 1(1): 1-20.

2.- Arnaoutakis D, Kim Y, Pulitano C, Zaydfudim V, Squires MH, Kooby D, et al. Management of Biliary Cystic Tumors: A Multi-Institutional Analysis of a Rare Liver Tumor. Ann Surg 2014;0(0):1-7.

3.- Nakayama Y, Kato Y, Okubo S, Takahashi D, Okada R, Nishida Y, et al. A case of mucinous cystic neoplasm of the liver: a case report. Surgical Case Reports 2015; 1(9):1-5.

4.- Lee CW, Tsai HI, Lin YS, Wu TH, Yu MC, Chen MF. Intrahepatic biliary mucinous cystic neoplasms: clinicoradiological characteristics and surgical results. BMC Gastroenterology 2015; 15(67):1-9.

5.- Valasek $M$, Yeh $M$. Mucinous Cystic Neoplasm of the Liver: Clinicopathologic Features and Differential Diagnosis case review. Pathol Case Rev 2014;19(1): 293-296.

6.- Mavilia M, Pakala T, Molina M, Wu G. Differentiating Cystic Liver Lesions: A Review of Imaging Modalities, Diagnosis and Management. J Clin Transl Hepatol 2018;6(2):208-216.

7.- Ariyarathna K, Anand BS, Lee JG, Mukherjee S. Hepatic Cystadenomas. Medscape, update 2016; 1(1):1-5.

Disponible en: https://emedicine.medscape.com/article/173056 overview\#showall.

8.- Soares K, Arnaoutakis D, Kamel I, Anders R, Adams R, Bauer TW, et al. Cystic Neoplasms of the Liver: Biliary Cystadenoma and Cystadenocarcinoma. J Am Coll Surg. 2014;218(1):119-128.
9.- Mortelé K, Ros P. Cystic Focal Liver Lesions in the Adult: Differential CT and MR Imaging Features. Radiographics 2001; 21(1):895-910.

10.- Lantinga M, Gevers T, Drenth J. Evaluation of hepatic cystic lesions. World J Gastroenterol 2013; 19(23): 3543-3554.

11.-Igor F. Current management of noninfectious hepatic cystic lesions: A review of the literatura. World J Hepatol 2013; 5(9): 462-469.

12.- Marcos R, Rodriguez A, Fernandez JM, Ramos P,Galván M, Gutierrez A, et al. Cistoadenomas hepatobiliares. Cir Esp 2006;79(6):375-8.

13.- Simo K, Mckillop L, Ahrens W , Martinie J , lannitti1 D, Sindram D. Invasive biliary mucinous cystic neoplasm: a review. HBP(Oxford).2012 ;14(11):725-740.

14.- Zen Y, Pedica F, Patcha VR, Capelli P, Zamboni G, Quaglia A, el al. Mucinous cystic neoplasms of the liver: a clinicopathological study and comparison with intraductal papillary neoplasms of the bile duct. Modern Pathology 2011; 24(1): 1079-1089.

15.-Lawrence P, Lalib Z, Aroori S, Bowles M, Stell D. Differentiating simple hepatic cysts from mucinous cystic neoplasms: Radiological features, cyst fluid, tumour marker analysis and multidisciplinary team outcomes. Dig Surg 2017;34(1):36-42

16.- Fragulidis G, Pantiora EV, Kontis EA, Primetis E, Polidoro A, Karvoun A, et al. Biliary Mucinous Cystic Neoplasm of the Liver with Ovarian Stroma and Elevated Serum and Cystic Fluid Cancer Antigen 19-9 Levels. Cureus2017; 9(11): 1-6.

Consulte la Versión Electrónica de la Revista:

Facultad de Medicina Humana Universidad Ricardo Palma

http://revistas.urp.edu.pe/index.php/RFMH
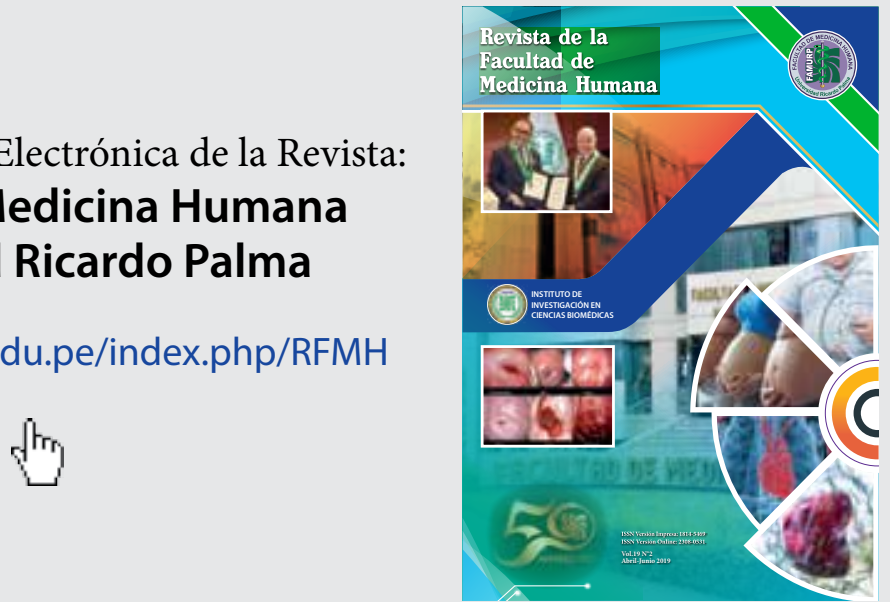